\title{
Diagnostic and Treatment Difficulties of Autoimmune Hepatitis on Systemic Lupus Erythematosus. Case Study
}

\author{
Egor Ignatiev $^{1}$, Diana Solovieva $^{2}$, Elena Popova $^{3, *}$ and Natalya Arkhipova ${ }^{4}$ \\ ${ }^{1}$ North-Eastern Federal University named after M.K. Ammosov, 678281, Russia \\ ${ }^{2}$ North-Eastern Federal University named after M.K. Ammosov, 678380, Russia \\ ${ }^{3}$ North-Eastern Federal University named after M.K. Ammosova, 677010, Russia \\ ${ }^{4}$ Republican Hospital № 1 - National Center of Medicine, 677010, Russia \\ ${ }^{*}$ Corresponding author. Email: popova.e.k@empl.s-vfu.ru
}

\begin{abstract}
On the example of case study, we present the features of the course of AIH on the background of SLE, as well as the difficulties of diagnosis and treatment. The study revealed an immune-mediated combination of autoimmune hepatitis with systemic lupus erythematosus, based on the association of autoimmune disorders with the main complex of human histocompatibility. The literature lacks data on the features of the course and treatment of comorbid patients with these pathologies. Autoimmune hepatitis on the background of systemic lupus erythematosus is characterized by early manifestation of the clinical picture, rapid progression of acute liver failure. The identification of immunological markers is diagnostically important; however, the morphological confirmation of the diagnosis is of paramount importance for deciding on the treatment. It should be borne in mind that AIH can proceed under the guise of other clinical signs for several years and can be mistakenly regarded as SLE, rheumatoid arthritis. Treatment involves a high risk of developing hepatic encephalopathy in response to pathogenetic therapy.
\end{abstract}

Keywords: autoimmune disorders, AIH, SLE, autoimmune hepatitis, systemic lupus erythematosus, immune-mediated combination

\section{INTRODUCTION}

Autoimmune hepatitis (AIH) is an independent chronic liver disease that has various clinical phenotypes, an unknown etiology. The prevalence of $\mathrm{AIH}$ in most cases is observed among women with elevated gamma globulin, immunoglobulin $\mathrm{G}(\mathrm{IgG})$, the presence of autoantibodies (AT), as well as human leukocyte antigens (HLA) DR3 and DR4, morphological signs of periportal hepatitis and a favorable response to immunosuppression [1].

Autoimmune processes with impaired tolerance to their own liver cells are known to be crucial in pathogenesis. In clinical practice, timely diagnosis of AIH is difficult, which does not rarely lead to omissions in the possibility of timely therapy at the initial stages of the disease, as well as the development of other autoimmune or immunoimmune pathological diseases. Among them, there is an association of AIH with systemic lupus erythematosus (SLE).

AIH is often accompanied by concomitant autoimmune or immune-mediated diseases such as autoimmune thyroiditis, rheumatoid arthritis, type 1 diabetes mellitus, systemic lupus erythematosus, Sjögren's syndrome, celiac disease, idiopathic thrombocytopenic purpura. SLE is characterized by a genetic abnormality of $\mathrm{T}$ and $\mathrm{B}$ lymphocytes, which, in turn, synthesize cytotoxic antibodies. The resulting immune complexes containing antinuclear antibodies (ANA) stimulate Th1-induced inflammation of connective tissues. According to the researches, it is known that AIH with HLA-DR4 is predominantly distributed in the countries of Southeast Asia, Japan, the 
clinical course of the disease is characterized by frequent systemic manifestations [2-4].

The association of autoimmune hepatitis with SLE suggests the presence of common links in the pathogenesis of these diseases. There is insufficient data in the literature on the features of the course and treatment of comorbid patients. The aim of our study is to analyze the clinical case of the combined autoimmune pathology of AIH and SLE, features of the administration, diagnosis and treatment of the disease.

\section{CASE STUDY}

Patient V. born in 1968, was in hospital treatment in January 2020 in the gastroenterological department of the Yakutsk Republic Clinical Hospital with complaints of pain, severity in the right subcostal area, jaundice of the skin and mucous membranes, weakness, reduced body weight, swelling on the lower limbs.

The patient has aggravated heredity from the mother, who suffered from severe liver failure on the background of liver cirrhosis of an unknown etiology.

Since 2010, the patient was observed in the district policlinic, with complaints of fever, arthralgia, a symptom of a "butterfly" on her face, a trophic ulcer in the left tibia. Taking into account the clinical picture, the detection of specific Le-cells in the blood made it possible to verify the diagnosis of SLE, Raynaud's syndrome. Steroid therapy was prescribed, followed by persistent remission for 5 years. From 2014 - 2018 the patient did not make complaints characteristic of SLE. During this period, the patient did not take steroid drugs.

In May 2017, the patient was operated on meningioma. A year later, in 2018, type 2 diabetes mellitus was diagnosed. During the medical examination in May 2019, an increase in the level of alpha-fetoprotein oncomarker (AFP) to $48.1 \mathrm{IU} / \mathrm{ml}$ was revealed (Table 1). In blood serum, transaminases and pigment exchange rates were within reference values. Due to the increase in AFP levels to $159 \mathrm{IU} / \mathrm{ml}$ and a progressive decrease in body weight, the patient was sent to Republic hospital No. 1 of Yakutsk, in order to exclude carcinogenic pathology. According to the results of the survey, oncopathology was excluded.

In early January 2020, the patient was hospitalized with suspected acute cholecystitis and mechanical jaundice with complaints of increasing pain in the right sucostal area, jaundice of the skin, mucous membranes, diarrhea and changes in biochemical blood analysis (13.01.20) in the form of an increase in total bilirubin $182.1 \mu \mathrm{mol} / \mathrm{l}$, direct bilirubin - $126 \mu \mathrm{mol} / \mathrm{l}, \mathrm{ShF}-513$ $\mathrm{U} / 1$ and a slight increase in ALT to $88 \mathrm{U} / 1$ (Table 2. A). Due to severe condition, the patient was urgently hospitalized in the Republic Hospital No. 2 of Yakutsk after 7 days. During the stay in the hospital, there was a negative dynamics in the patient's condition in the form of an increase in general weakness and jaundice. Biochemical blood analysis from 15.01.20: gammaglutamyl transpeptidase (GGT) - $77.5 \mathrm{U} / \mathrm{l}$, total bilirubin $165.1 \mu \mathrm{mol} / \mathrm{l}$, direct bilirubin $149.6 \mu \mathrm{mol} / \mathrm{l}$, ALT 417.5 U/l, ACT 1533.8 U/l, albumin $20.1 \mathrm{~g} \mathrm{A,} \mathrm{B).}$ Coagulogram: Quick's value $28 \%$, MNO 2.45, prothrombin time $30.50 \mathrm{sec}$, fibrinogen $2.5 \mathrm{~g} / \mathrm{l}$, aPTT 47.7 sec. Oncomarkers: antigen of adenogenic cancer SF 19-9, alpha-fetoprotein 201.6; cancer-embryonic antigen 6.8. In order to carry out differential diagnostics, autoimmune markers were determined: AT to mitochondrial antigens (AMA-M2) 0.2; No anti-nucleic antibodies (ANA) were detected. According to the ultrasound of the abdominal organs, computed tomography with contrast showed signs of hepatosplenomegaly, diffuse changes in parenchyma, signs of bile stone disease (BSD). With esophagogastroduodenoscopy (EGDS), cardiac insufficiency, reflux esophagitis, erosive-hemorrhagic gastritis and varicose veins of the lower third of the esophagus of degree I were detected. According to the abdominal MRI from 24.01.2020, specifics of the gallbladder were found, minor dilatation of choledoch, no signs of choledocholytiasis were found. There are signs of cirrhosis of the liver in the form of diffusely heterogeneous fine-knot degeneration of the liver parenchyma, a small amount of free fluid is observed in the abdomen.

Glucocorticosteroid (GCS) therapy was initiated at a dose of $60 \mathrm{mg} / \mathrm{day}$. On the background of taking GCS the patient in the first days had a decrease in blood serum transaminases: ALT 195.8 U/L, ACT 347.7 U/L, alkaline phosphatase $315.2 \mathrm{U} / \mathrm{L}$, total bilirubin up to $135.2 \mu \mathrm{mol} / \mathrm{L}$, direct bilirubin $97.8 \mu \mathrm{mol} / \mathrm{L}, \mathrm{CRP} 12.93$ $\mathrm{mg}$. On the fourth day of GCS therapy, signs of progression of hepatic encephalopathy suddenly appeared, disorientation, somnolence, left-sided hemiparesis, impaired hemitip sensitivity on the left were observed. In this regard, immunosuppressive therapy of GCS was canceled, followed by prescribing Ornithine infusions of $20 \mathrm{ml}$, Lactulose at a dose of $30 \mathrm{ml}$ per day, Ursodeoxycholic acid of $250 \mathrm{mg}$ and cleansing edema. Etamzilate $4 \mathrm{ml}$ and Vicasol $10 \mathrm{mg} / \mathrm{ml}$ were prescribed to correct blood coagulopathy. On the second day after the start of detoxification therapy and withdrawal of GCS, the patient's condition improved, but general weakness, rapid fatigue and weakness in the left limbs remained. At the conclusion of the medical consultation, it was decided not to prescribe targeted therapy and maintenance dosage of GCS, due to the high risk of repeated progression of hepatic encephalopathy. In mid-February 2020 the patient was discharged in satisfactory condition.

For the purpose of differential diagnosis, immunological analysis was carried out to identify AIH markers (Table 4), according to the results of which the diagnosis of AIH type 1 was verified. 
In April 2020 during plan hospitalization for treatment correction due to verification of the diagnosis of $\mathrm{AIH}$, the patient progressed hepatic encephalopathy, provoked by the onset of hormone therapy and stool retention for 4 days. Hormone therapy was prescribed for only two days - prednisolone $90 \mathrm{mg} /$ day, then continued treatment with Ornithine, Ursodeoxycholic acid.
Table 1. Blood Test for alpha fetoprotein

\begin{tabular}{|l|l|l|l|}
\hline Onkomarker & 08.05 .2019 & 22.11 .2019 & 20.01 .2020 \\
\hline alpha fetoprotein & 48.1 & 159 & 201.6 \\
\hline
\end{tabular}

Table 2a. Biochemical Blood Test

\begin{tabular}{|c|c|c|c|c|c|c|c|c|c|}
\hline Biochemical blood tests & $\begin{array}{l}22.11 \\
2019\end{array}$ & $\begin{array}{l}04.01 \\
2020\end{array}$ & $\begin{array}{l}13.01 \\
2020\end{array}$ & $\begin{array}{l}15.01 \\
2020\end{array}$ & $\begin{array}{l}17.01 \\
2020\end{array}$ & $\begin{array}{l}20.01 \\
2020\end{array}$ & $\begin{array}{l}24.01 . \\
2020\end{array}$ & $\begin{array}{l}27.01 \\
2020\end{array}$ & $\begin{array}{l}30.02 \\
2020\end{array}$ \\
\hline GGT unit/l & & & & 77.5 & 68.0 & 54.0 & & 53.0 & 52.0 \\
\hline Bilirubin: total, $\mu \mathrm{mol} / \mathrm{l}$ & 15.0 & 124.0 & 182.1 & 1651 & 175.0 & 201.0 & 135.2 & 161.0 & 131.0 \\
\hline Direct, $\mu \mathrm{mol} / \mathrm{l}$ & & 58.6 & 126 & 1496 & 155,4 & 169.6 & 97.8 & 136.1 & 112.1 \\
\hline ALT unit/l & 9.0 & 5.7 & 88 & 417 & 357.2 & 299.1 & 195.8 & 173.9 & 152.4 \\
\hline ACT units/l & & & & 1533.8 & 1321.9 & 981.4 & 347.7 & 397.4 & 366 \\
\hline Urea, $\mathrm{mmol} / \mathrm{L}$ & & & 2.3 & 2.6 & 2.9 & 2.8 & 3.4 & 3.6 & 3.9 \\
\hline Albumin, $g / l$ & & & & 20.1 & 24 & 29 & 32.6 & 38 & 39 \\
\hline Creatinine, $\mathrm{mmol} / \mathrm{L}$ & 93 & & 60 & 54 & 85.0 & 78.0 & 41.5 & 83.0 & 78 \\
\hline Cholesterol, mmol/L & & & 3.8 & 3.6 & 3.1 & 2.9 & 4.0 & 4.6 & 4.4 \\
\hline Alkaline phosphatase, unit/l & & 137 & 513 & 378 & 322 & 293 & 315.2 & 275 & 365 \\
\hline C-reactive protein, mg/l & & & & & 9.1 & 9.8 & 12.93 & 14.8 & 14.4 \\
\hline Total protein, $\mathrm{g} / \mathrm{l}$ & & & 75.7 & 75.6 & 74.2 & 75.1 & 76.7 & 84.4 & 84.4 \\
\hline Glucose, $\mathrm{mmol} / \mathrm{L}$ & 4.0 & & 3.09 & 4.30 & 4.67 & 4.45 & 4.9 & 3.85 & 6.25 \\
\hline
\end{tabular}

Table 2b. Biochemical Blood Test

\begin{tabular}{|c|c|c|c|c|c|c|c|c|c|c|}
\hline Biochemical blood tests & $\begin{array}{l}04.02 \\
2020\end{array}$ & $\begin{array}{l}11.02 \\
2020\end{array}$ & $\begin{array}{l}03.03 \\
2020\end{array}$ & $\begin{array}{l}31.03 . \\
2020\end{array}$ & $\begin{array}{l}15.04 \\
2020\end{array}$ & $\begin{array}{l}21.04 . \\
2020\end{array}$ & $\begin{array}{l}13.05 \\
2020\end{array}$ & $\begin{array}{l}29.06 \\
2020\end{array}$ & $\begin{array}{l}17.08 \\
2020\end{array}$ & $\begin{array}{l}05.10 \\
2020\end{array}$ \\
\hline GGT unit// & 52.0 & 58.0 & & & & 32.0 & & 37 & & \\
\hline Bilirubin: total, $\mu \mathrm{mol} / \mathrm{l}$ & 117.0 & 136.0 & 113.6 & 80.6 & 100.0 & 68.0 & 57.9 & 29.5 & 28.1 & 16.9 \\
\hline Direct, $\mu \mathrm{mol} / /$ & 99.5 & 115.9 & 91.9 & 72.2 & 36.0 & 53.9 & 33.4 & 21.1 & 8.4 & 3.5 \\
\hline ALT unit/l & 181.5 & 178.4 & 117.2 & 86.2 & 68 & 37.8 & 49 & 24.7 & 39 & 53 \\
\hline ACT units/l & 455 & 436.4 & 341 & 277.5 & 99 & 66.4 & 131 & 76.9 & & \\
\hline Urea, $\mathrm{mmol} / \mathrm{L}$ & 3.6 & 3.8 & 4.2 & 3.5 & 7.6 & 4.8 & & & 5.62 & 5.62 \\
\hline Albumin, $\mathrm{g} / \mathrm{l}$ & 36 & 36 & 26.8 & 20.8 & 23 & 30 & 31 & 29.8 & & \\
\hline Creatinine, $\mathrm{mmol} / \mathrm{L}$ & 77.0 & 84.0 & 77 & 65 & 91.0 & 78.0 & 73 & & 74.5 & 83 \\
\hline Cholesterol, mmol/L & 4.1 & 3.9 & 2.36 & 2.63 & & 3.0 & & 4.2 & 3.66 & 5.1 \\
\hline Alkaline phosphatase, unit/l & 255 & 272 & & 250 & & 196 & & & 765 & \\
\hline C-reactive protein, mg/l & 14.8 & 11.4 & & & & 20.4 & 5 & & & \\
\hline Total protein, g/l & 81.5 & 84.4 & & 74.4 & 75.0 & 72.0 & 74 & 69.4 & 64.5 & 73 \\
\hline Glucose, $\mathrm{mmol} / \mathrm{L}$ & 7.93 & 4.53 & 4.04 & 3.72 & 10.9 & 4.25 & & 6.34 & 5.01 & 5.51 \\
\hline
\end{tabular}

Table 3. Immunological blood test: SLE markers from 19.12.2019

\begin{tabular}{|l|l|}
\hline Tests & Result \\
\hline AT to native (double spears.) DNA IgG & 118.80 \\
IgE (total) & $\mathrm{ME} / \mathrm{ml}$ \\
\hline IgE (total) & $248 \mathrm{ME} / \mathrm{ml}$ \\
\hline C3 complement component & $0.86 \mathrm{~g} / \mathrm{l}$ \\
\hline
\end{tabular}




\begin{tabular}{|l|l|}
\hline S4 complement content & $0.13 \mathrm{~g} / \mathrm{l}$ \\
\hline Anti-Nucleic Antibodies, IgG Screening (ANA) & Positive \\
\hline
\end{tabular}

Table 4. AIH markers dated 23.03.2020

\begin{tabular}{|l|l|}
\hline Indicators & Result \\
\hline IgG & $30.67 \mathrm{~g} / \mathrm{I}$ \\
\hline Anti-Nucleic Antibodies, IgG Screening (ANA) & Positive \\
\hline Smooth Muscle AT (SMA), IgG+A+M & $1: 160$ titre \\
\hline AT to liver and kidney microsomes, IgG+A+M & $<1: 40$ titre \\
\hline
\end{tabular}

As a result, the following diagnosis was verified:

Main: Cirrhosis of the liver class B according to Child Pugh, in decompensation stage in the outcome of autoimmune hepatitis type 1, high degree of activity.

Complications: Portal hypertension, varicose veins of the esophagus 1 stage. Coagulopathy. Chronic liver failure. Hepatic encephalopathy type C, class II, repeated, provoked (stool retention).

Concomitant: SLE, chronic course, activity 1 , antinuclear AT, AT to two-spiral DNA, a history of erythema on the face in the form of a "butterfly," fever, oral ulcers, polyarthritis. Type 2 diabetes mellitus, subcompensated. Gallstone disease. Chronic calculous cholecystitis outside exacerbation. Condition after bifrontal bone-plastic craniotomy of microsurgical removal of falx meningioma from 15.05.2017.

\section{DISCUSSION}

Based on the mentioned above, the diagnosis of SLE in this patient $\mathrm{B}$. was diagnosed before the verification of AIH. It can be assumed that these diseases are characterized by the presence of common links of pathogenesis. Thus, the association of autoimmune disorders with the main complex of human histocompatibility is traced. It is known that most autoimmune diseases, in particular SLE and AIH, are associated with the presence of the following antigens in the HLA phenotype: DRB1, DR2 and DR3, DR4 respectively [5, 6]. Presumably, the genes of the HLA system take part in the selection of T-lymphocytes, the process of which is disrupted when certain alleles are present, as a result of which there is no elimination of sensitized T-lymphocytes to autoantigens. Such a connection is important when diagnosing AIH: the presence of SLE in the case history indicates impaired immunity tolerance associated with HLA complex.

According to the researches, often one of the options for development of AIH is observed by the manifestation of extrahepatic manifestations, fever, arthralgia for several years and can be erroneously regarded in the form of SLE, rheumatoid arthritis [2-4, 7]. The course of AIH in association with SLE is characterized by the following features: the manifestation of clinical manifestations of AIH with acute increase in laboratory indicators occurs in a shorter period of time.

The duration of the presented case study of patient B. was 10 months. During this period, the patient had a pronounced clinical symptomatology of hepatic encephalopathy, which fastly developed on the background of treatment with glucocorticosteroids. There is evidence in the literature that in $43 \%$ of cases $\mathrm{AIH}$ is formed within three years in the absence of cirrhosis treatment [8]. But in this patient, in the presence of pathogenetic therapy, cirrhosis formed in less than a year. Perhaps this is due to the variability in the course of the disease, associated with features of antigenic histocompatibility, with the role of a transcription factor, designated as type 1 autoimmune regulator, which causes a predisposition to the development of AIH.

From the above anamnesis and clinical picture, positive dynamics of the disease can be clearly traced against the background of UDCA therapy, despite the absence of pathogenetic therapy.

The efficacy and safety of the use of UDCA B in the treatment of liver cirrhosis in the outcome of AIH still remains and is a subject of discussion in the medical community, but we would like to emphasize that UDCA positions itself as one of the highly effective drugs in the treatment of diseases of the hepatobiliary system according to domestic and international recommendations.

According to the literature, AIH treatment is carried out in a combination of GCS and UDCA, due to the peculiarity of the molecular structure - due to its structural similarity with GCS, it modulates the activation of the nuclear receptor of steroid drugs, thereby leading to the potentiation of the effect

\section{CONCLUSION}

The above case study shows the course of AIH on the background of SLE, which is characterized by rapid progression and early clinical manifestations, as well as a pronounced manifestation of hepatic encephalopathy. Final verification of AIH type 1 requires a liver biopsy with the identification of a characteristic morphological picture of AIH - lobular hepatitis with bridge-like or massive necrosis, emperipolesis, hepatocytic rosettes.

If the patient has signs of liver damage with impaired tolerance of the immune system, it is recommended to exclude AIH. In the diagnosis of AIH, the first sign of alertness is an increase in ACE oncomarker titers, often detected by screening, in patients with autoimmune diseases. It should be noted that AIH can occur under the mask of other clinical signs for several years and can be erroneously regarded in the form of SLE, rheumatoid arthritis [2-4]. 
When diagnosing AIH of high activity with positive markers, early initiation of pathogenetic therapy is necessary, despite the lack of results of morphological analysis of the liver (biopsy). But the risk of developing hepatic encephalopathy in response to GCS treatment must be considered. An alternative therapy may be the use of genetically engineered biologics and liver transplantation.

\section{REFERENCES}

[1] EASL, J. Hepatol. 5 (2015) 111-112.

[2] V.T. Ivashkin, A.O. Bueverov, M-Vesti 112 (2011).

[3] V.T. Ivashkin, M.V. Mayevskaya, MED pressinform, 2017, pp. 75-84.

[4] C.P Strassburg, M.P Manns, J. Hepatol. 45 (2006) 480-482.

[5] P.N. Kravchenko, E.K. Oleynik, Works of the Karel. Sci. center RAS 12 (2015) 3-22.

[6] S.D. Podymova, Experim. and clin. Gastroenterol. 8 (2017) 33-44.

[7] M.P Manns et al., Diagnosis and management of autoimmune hepatitis, Hepatol. 8 (2010) 51.

[8] E.K. Popova, N.S. Arkhipova, E.A. Ignatiev et al., Yakutsk med. J. 1(73) (2021) 119-122. 